\title{
Experimental Observation of Plasma Wake-Field Acceleration
}

\author{
J. B. Rosenzweig, D. B. Cline, ${ }^{(a)}$ B. Cole, ${ }^{(b)}$ H. Figueroa, ${ }^{(c)}$ W. Gai, R. Konecny, J. Norem, \\ P. Schoessow, and J. Simpson \\ High Energy Physics Division, Argonne National Laboratory, Argonne, Illinois 60439
}

(Received 21 March 1988)

\begin{abstract}
We report the first experimental test of the physics of plasma wake-field acceleration performed at the Argonne National Laboratory Advanced Accelerator Test Facility. Megavolt-per-meter plasma wake fields are excited by a intense $21-\mathrm{MeV}$, multipiscosecond bunch of electrons in a plasma of density $n_{e}=10^{13} \mathrm{~cm}^{-3}$, and probed by a low-intensity $15-\mathrm{MeV}$ witness pulse with a variable delay time behind the intense bunch. Accelerating and deflecting wake-field measurements are presented, and the results compared to theoretical predictions.

PACS numbers: $52.75 . \mathrm{Di}, 52.40 . \mathrm{Mj}$
\end{abstract}

The plasma wake-field accelerator (PWFA) concept has been the subject of much theoretical discussion in the last few years, in large part because of the possibility of the achievement of ultrahigh accelerating gradients for high-energy-physics use through this scheme. The predictions of the linear fluid theory and computer simulation ${ }^{1-3}$ in two dimensions have identified certain issues for experimental study and verification. These include the fundamental excitation of the electron plasma waves with their associated electrostatic fields, the electromagnetic self-pinching of a high-intensity driving beam, and the dependence of these processes on plasma and beam characteristics. The testing of these issues is naturally suited to the properties of the Argonne National Laboratory Advanced Accelerator Test Facility ${ }^{4}$ (AATF).

A schematic of the AATF layout is shown in Fig. 1. The high-intensity $21-\mathrm{MeV}$ driving-beam pulse traverses the upper beam line. The low-intensity, $15-\mathrm{MeV}$ witness beam, which is created by our degrading a portion of the driver on a carbon target just prior to the facility entrance, is transported through the lower beam line. The witness leg contains an adjustable "trombone" section
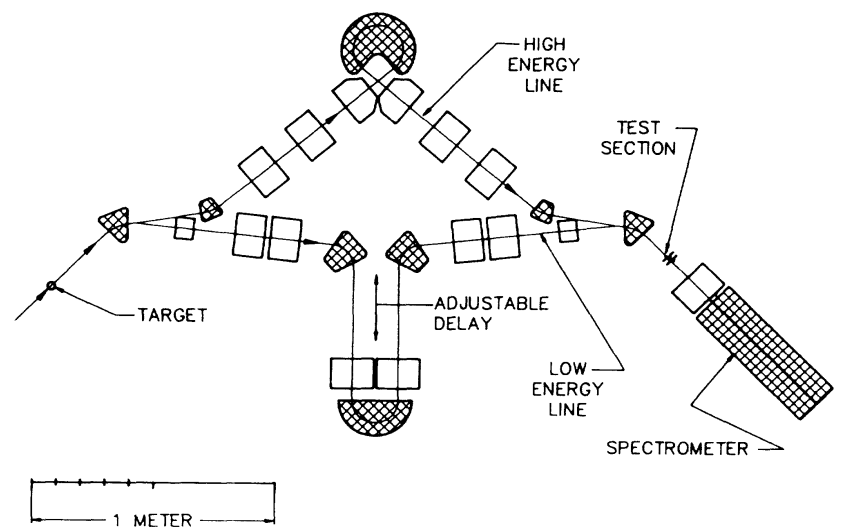

FIG. 1. Schematic of Argonne National Laboratory AATF layout. which allows variation of the delay time between driver and witness. The two beams are recombined at the end of the facility and pass through the experimental section. Both beams are analyzed for energy spectra and transverse deflections by a high-resolution, broad-range spectrometer. The high intensity and short pulse length of the facility driving beam make it useful for driving plasma waves and their strong wake fields. However, the crucial unique experimental tool that the AATF possesses is its witness pulse, which allows the probing of the plasma wake fields at arbitrary positions behind the driver. The operational characteristics of the AATF are described in greater detail in Ref. 4.

The two-dimensional linear theory of plasma wake fields, as formulated by Chen, ${ }^{1}$ provides a simple model for the calculation of expected wake fields in a PWFA experiment. This model has been used in the optimization of the experimental parameters to match the beam characteristics at the AATF. These may be summarized as follows: beam length, as measured by a 2-psec-resolution streak camera, $\sigma_{z}=2.4 \mathrm{~mm}$; beam width $\sigma_{r}=2.4$ $\mathrm{mm}$; and total driver-beam charge $2-3 \mathrm{nC}$. The driverand witness-beam dimensions are approximately equal, with the full width at half maximum of their respective density distributions in $z$ and $r$ defined to be equal to $2 \sigma$.

According to theory, plasma wake fields inside a driving beam are both electrostatic and electromagnetic. These fields have two effects in general-deceleration and focusing of the beam. On the other hand, the wake fields left behind the bunch are purely electrostatic oscillations which are sinusoidal at the plasma frequency $\omega_{p}=\left(4 \pi e^{2} n_{e} / m_{e}\right)^{1 / 2}$, where $n_{e}$ and $m_{e}$ are the equilibrium plasma density and the electron mass, respectively. The phase velocity of the waves is the beam velocity $v_{b}$, and thus the oscillation wave number and wavelength are $k_{p}=\omega_{p} / v_{b}$ and $\lambda_{p}=2 \pi / k_{p}$.

A straightforward way of understanding the expected wake fields is to note that the longitudinal wake-field amplitude can be expressed in terms of the driver charge $Q$ and two geometric factors. An infinitesimally short 
(disk-shaped) driving beam of surface charge density $\Sigma$ generates a maximum longitudinal wake field of $W_{m}$ $=4 \pi e \Sigma \propto Q / \sigma_{r}^{2}$. However, if the plasma wavelength is larger than the beam width, the fields spread out and become predominantly radial. The maximum longitudinal wake field is reduced by a factor $\eta_{r}\left(k_{p} \sigma_{r}\right)$, which approaches unity if $k_{p} \sigma_{r} \ll 1$ and zero if $k_{p} \sigma_{r} \gg 1$. The other geometric factor concerns the longitudinal plasma response. If $K_{p} \sigma_{z}$ is not small compared to 1, the plasma has time to respond to the beam charge and moves to neutralize the beam. This lessens the coupling of the beam charge to the wave and reduces the maximum longitudinal wake field. For Gaussian beam profiles, this factor is $\eta_{z}\left(k_{p} \sigma_{z}\right)=\exp \left[-\left(k_{p} \sigma_{z}\right)^{2} / 2\right]$.

It is clear from the discussion above that the maximum longitudinal wake fields are generated if $k_{p} \sigma_{z}$ $\ll 1 \ll k_{p} \sigma_{r}$, which means a beam much shorter than it is wide. The beam in this experiment is approximately round $\left(\sigma_{z} \simeq \sigma_{r}\right)$ and is thus not quite optimum. The excitation efficiency function $\eta_{t}=\eta_{z} \eta_{r}$ for our case has a maximum value of about 0.45 , at a point where $k_{p}$ would indicate use of a plasma density of $n_{e}=8 \times 10^{12} \mathrm{~cm}^{-3}$ for our beam dimensions. We may consider this an optimum point in plasma density to observe large accelerating gradients in the PWFA. Experimentally, there are two complications in this case over a disk-shaped beam: The transverse fields are larger relative to the longitudinal fields, and, with a witness-beam length not small compared with a plasma wavelength, the resolution of the measurement is degraded.

The dc plasma source used for the PWFA experiment is a hollow-cathode arc constructed at the University of Wisconsin. The plasma length $L$ is $20-35 \mathrm{~cm}$, and density $n_{e}$ is variable about our optimum working point, approximately $(0.7-7.0) \times 10^{13} \mathrm{~cm}^{-3}$, with an electron temperature of 2-8 eV. An axial magnetic field 50-700 $\mathrm{G}$ is applied to confine the plasma column. The feed gas

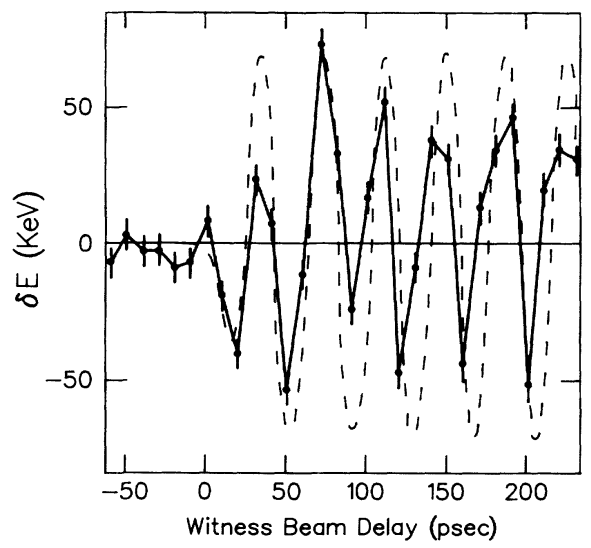

FIG. 2. Scan 1: Witness-beam energy-centroid change $\delta E$ vs time delay behind driver. Total driver-beam charge $Q=2.1$ nC; plasma parameters $L=28 \mathrm{~cm}$ and $n_{e}=8.6 \times 10^{12} \mathrm{~cm}^{-3}$. Theoretical predictions are given by the dashed line. is argon, and the background pressure is limited to approximately $1-2 \mu \mathrm{m}$ by use of a $750-1 / \mathrm{sec}$ turbomolecular pump. Plasma diagnostics include electrostatic probes and microwave interferometry.

Detection of both the witness- and driver-beam profiles at the focal plane of the spectrometer, as well as at the beam-position monitors, is accomplished by closed-circuit television monitoring of positionable phosphor screens. The video images are digitized through use of a frame grabber and stored on disk for off-line analysis. The witness-beam data are complicated by the large and rapidly fluctuating background of light generated by the driver-beam halo which precludes a simple subtraction of a background frame from the data. Instead, an algorithm is employed which extracts the majority of the witness-beam spot from the rest of the image, and calculates the resulting centroids of the image distribution in the dispersive and deflection planes. Background base-line shifts due to changing witnessbeam transmission optics are then subtracted.

Witness-beam delay scans, displaying beam-energy centroid as a function of the time delay between driver and witness beam, are shown in Figs. 2 (scan 1) and 3 (scan 2). The dashed lines indicate the predictions of the simple theoretical model, plotted for comparison to the data. The theoretical predictions include the experimental effects of the resolution of the witness beam, which we discuss below. These effects make the predictions approximate because of the shot-to-shot fluctuation of the beam length and charge measurements. All theoretical predictions consistent with these uncertainties would form a band about the dashed line shown in each figure. Only the central prediction is shown for clarity. Ahead of the driver, we observe small fluctuations in the energy centroid due to background and linac fluctuations. The centroid then lowers while passing through the region near zero delay, as it feels the decelerating

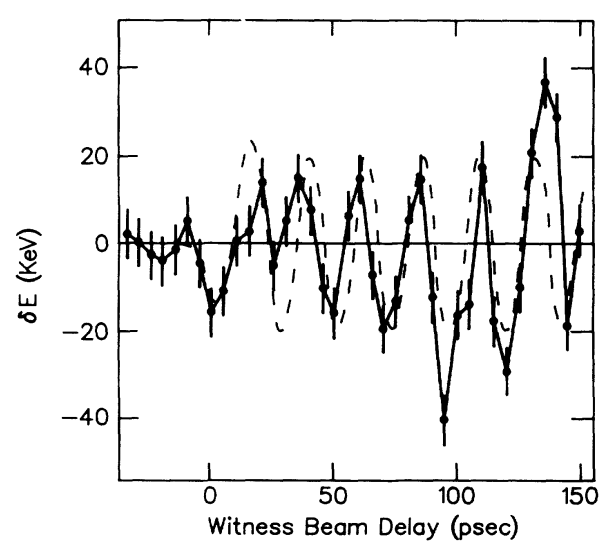

FIG. 3. Scan 2: Witness-beam energy centroid change $\delta E$ vs time-delay total driver-beam charge $Q=2.1 \mathrm{nC}$; plasma parameters $L=33 \mathrm{~cm}$ and $n_{e}=2.3 \times 10^{13} \mathrm{~cm}^{-3}$. Theoretical predictions are given by the dashed line. 
wake on the driver. Behind the driver we observe oscillations characteristic of the plasma wake fields, harmonic with the plasma frequency. These oscillations persist for many wavelengths and maintain a nearly constant frequency. In both scans the driver-beam charge, measured by use of the spectrometer as a Faraday cup, was determined to be $2.1 \mathrm{nC}$, with shot-to-shot fluctuation of approximately $5 \%$. The plasma characteristics were as follows: For scan $1, L=28 \mathrm{~cm}$ and $n_{e}=8.6 \times 10^{12} \mathrm{~cm}^{-3}$, and for scan $2, L=33 \mathrm{~cm}$ and $n_{e}=2.3 \times 10^{13} \mathrm{~cm}^{-3}$. The densities quoted above were deduced from fast-Fouriertransform calculation of the oscillation frequency. They agree remarkably well with electrostatic probe measurements, as shown by the comparison in Fig. 4, which gives a relative error of less than $20 \%$.

The maximum accelerating wake-field amplitude $W_{m}$ can be calculated from the maximum centroid energy excursions $\delta E_{m}$. We must first average over the longitudinal wake field felt by different parts of the witness-beam spatial distribution, and divide by the resulting resolution function. This function can be divided into two factors, a radial resolution function $R_{r} \simeq \sigma_{r, d}^{2} /\left(\sigma_{r, w}^{2}+\sigma_{r, d}^{2}\right)$, where $\sigma_{r, d(w)}$ is the radial beam size of the driver (witness), and a longitudinal resolution function $R_{l}$ $=\exp \left[-\left(k_{p} \sigma_{z}\right)^{2} / 2\right]=\eta_{l}$. For both scans the radial resolution $R_{r}$ is about 0.5 , but with the higher density in scan 2 one obtains less longitudinal resolution because of the shortening of the plasma wavelength. For the beam and plasma characteristics of scan $1, R_{l}=0.43$, and for scan $2, R_{l}=0.11$. The maximum wake field is thus given by the expression $W_{m}=\delta E_{m} / R_{r} R_{l} L$, which gives $W_{m} \simeq 0.94 \pm 0.27 \mathrm{MeV} / \mathrm{m}$ for scan 1 and $1.60 \pm 0.63 \mathrm{MeV} / \mathrm{m}$ for scan 2. The uncertainty in the determination is due mainly to the $20 \%$ uncertainty in the beam length (as determined by streak-camera measurements) from shot

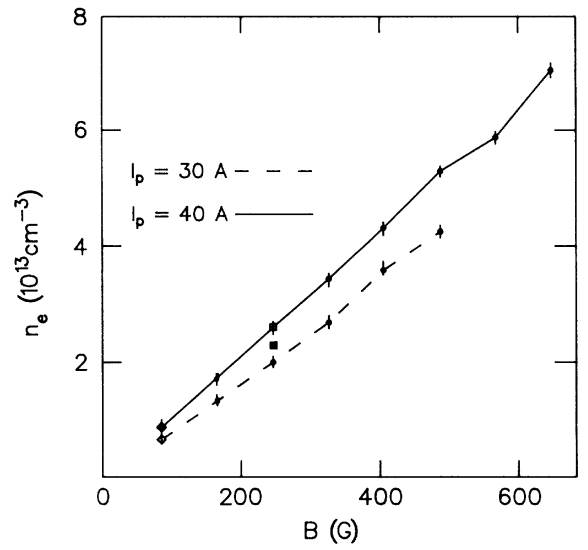

FIG. 4. The plasma electron density $n_{e}$ from probe measurements for various running conditions (arc current $I_{p}$ and applied axial magnetic field $B$ ) of the arc source. The filled symbols show the density as determined from $\lambda_{p}$, and open symbols are the corresponding probe measurements: scan 1, diamond symbols; scan 2 , square symbols. to shot, which strongly affects the calculated wake-field amplitudes through the quickly varying longitudinal resolution function. The agreement with the simple theoretical model is quite good, as indicated in Figs. 2 and 3 .

Scanning further back in delay shows a decay in amplitude of the measured energy-centroid shifts. Since the plasma is not collisional $\left(\omega_{p} \gg v_{e-i}\right.$, the collision frequency), we attribute this decay to the longitudinal density gradient along the plasma column. The phase delay between the two beams is related to the time delay by $\Delta \phi=\omega_{p}(z) \Delta t$, so that a variation in the plasma density changes the phase delay more dramatically at larger time delays. These larger changes in the phase delay cause the total wake fields integrated by the witness beam to be diminished. If we assume that the plasma density gradient along the column is small and constant, the total density variation $\delta n_{e} / n_{e}$ in the plasma can be related to the observed damping of the measured wakefield amplitude $\delta W_{m} / W_{m}$ by

$$
\frac{\delta n_{e}}{n_{e}} \simeq \frac{2 \delta \omega_{p}}{\omega_{p}}=\frac{4}{\omega_{p} \Delta \tau}\left(3 \frac{\delta W_{m}}{W_{m}}\right)^{1 / 2}
$$

For a scan in which we observed a degradation in amplitude $\delta W_{m} / W_{m} \simeq 0.62$ after ten plasma wavelengths of delay, we deduce a density variation of about $9 \%$. This also is consistent with electrostatic probe measurements.

The transverse wake fields in plasmas are of great interest because of their possible application as a final focusing scheme for linear colliders. ${ }^{1,5}$ Transverse deflections in the witness-beam nonbend-plane position centroid were observed when the witness beam and driver were not collinear. One such scan is shown in Fig. 5 , which plots the position centroid versus delay time.

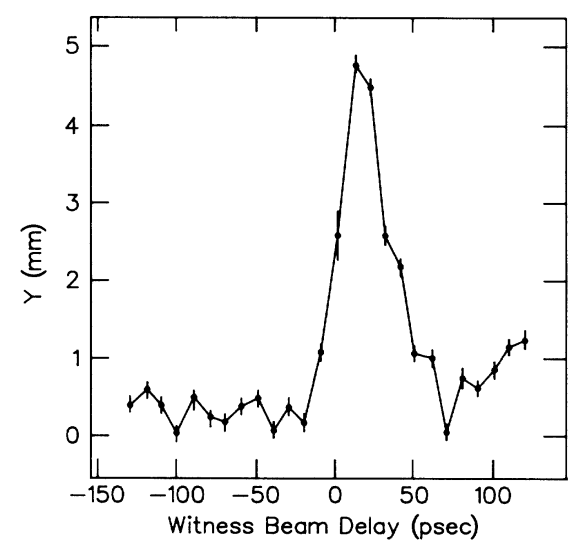

FIG. 5. Witness-beam centroid position $Y$ in the nonbend plane of the spectrometer vs delay, for off-axis injection of witness beam. The large deflection near zero delay is expected from theory, because of the self-focusing magnetic wake fields at the driver. For this scan $Q=2.2 \mathrm{nC}, L=33 \mathrm{~cm}$, and $n_{e}$ $=3.4 \times 10^{-13} \mathrm{~cm}^{-3}$. 


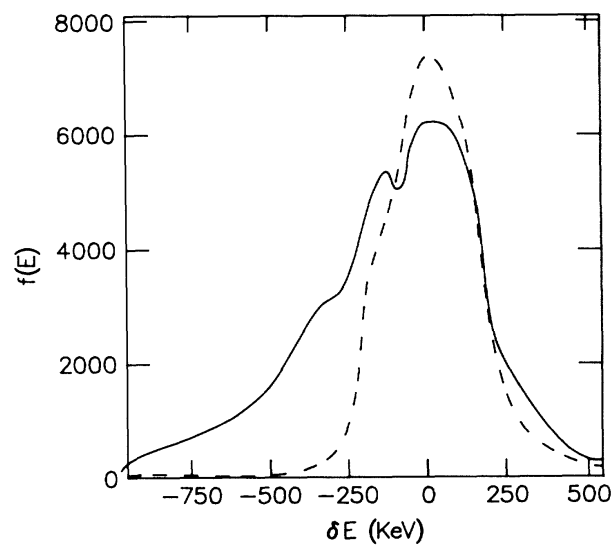

FIG. 6. Driver-beam energy spectrum for $Q=2.5 \mathrm{nC}$, $L=28 \mathrm{~cm}$, and $n_{e}=2.9 \times 10^{-13} \mathrm{~cm}^{-3}$ plotted with a solid line, along with the case of no plasma present plotted with a dashed line.

For this scan the driver beam charge is $2.2 \mathrm{nC}$, the plasma length is $33 \mathrm{~cm}$, and the plasma density is $n_{e}$ $=3.4 \times 10^{-13} \mathrm{~cm}^{-3}$. The deflection is quite strong at zero delay, where the driver magnetic fields provide a strong pinching force. The plasma excitation efficiency is very low for this case because of the high plasma density, and so the wake fields are expected to be nonnegligible only inside the driver, as is observed. The 5-mm maximum position change on the focal plane corresponds to approximately $2.5 \mathrm{mrad}$ of deflection, or $37.5 \mathrm{keV}$ of transverse kick, which is of the same order of magnitude as the acceleration amplitudes observed in the on-axis scans.

A typical driver-beam energy-loss spectrum is shown in Fig. 6, with a comparison to the case of no plasma present. Qualitatively, we observe the most total driver energy loss at lowest plasma density, which is expected, as the total energy loss should scale as $\eta_{r} \eta_{l}^{2}$. However, we are unable to quantify the total energy loss well at present, because the self-pinching of the decelerating electrons deflects a portion of the beam distribution off the focal-plane detectors. This is further evidence, of course, for the strong focusing wake fields inside the driver beam.

In conclusion, we have performed a direct measurement of plasma wake fields by accelerating an injected witness-beam pulse in the wake of an intense driverbeam pulse in plasma. We have also demonstrated the existence of strong transverse wake fields using the witness beam. Future experimental work at the AATF will concentrate on increasing the driver current and shortening the pulses to drive higher wake-field amplitudes and improve the longitudinal resolution. Beyond this goal, we plan to study the effects of pulse shaping on transformer ratio, ${ }^{6}$ and interesting nonlinear effects in larger-amplitude plasma waves. ${ }^{7,8}$

The authors would like to acknowledge valuable contributions for F. Mills, J. Maclachlan, J. Detra, P. Sealy, A. Hershcovitc, and the University of Wisconsin Plasma Physics Group. This work was supported by the U.S. Department of Energy, Division of High Energy Physics, Contract No. W-31-109-ENG-38.

(a) Present address: Department of Physics, University of California at Los Angeles, Los Angeles, CA 90024.

(b) Permanent address: Department of Physics, University of Wisconsin-Madison, Madison, WI 53706.

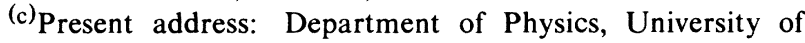
Southern California, Los Angeles, CA 90089.

${ }^{1} P$. Chen, Part. Accel. 20, 171 (1985).

${ }^{2}$ T. Katsouleas, Phys. Rev. A 33, 2056 (1986).

${ }^{3}$ R. D. Ruth, A. Chao, P. L. Morton, and P. B. Wilson, Part. Accel.17, 171 (1985).

${ }^{4}$ H. Figueroa, W. Gai, R. Konecny, J. Norem, P. Schoessow, and J. Simpson, Phys. Rev. Lett. 60, 2144 (1988).

${ }^{5}$ D. B. Cline, B. Cole, J. Rosenzweig, and J. Norem, in Proceedings of the 1987 IEEE Washington Particle Accelerator Conference, edited by E. Lindstrom and L. Taylor (IEEE New York, 1987), p. 154.

${ }^{6}$ K. L. F. Bane, P. Chen, and P. B. Wilson, IEEE Trans. Nucl. Sci. 32, 3524 (1985).

${ }^{7}$ R. Keinigs, M. Jones, and J. J. Su, IEEE Trans. Plasma Sci. 15, 199 (1987).

${ }^{8}$ J. B. Rosenzweig, Phys. Rev. Lett. 58, 555 (1987). 